\title{
Small Nucleolar RNA Score: An Assay to Detect Formalin-Overfixed Tissue
}

\author{
Wim Ammerlaan,, Johanna Trouet,, Michael C. Sachs, ${ }^{1}$ Ping Guan, ${ }^{3}$ Latarsha Carithers, ${ }^{3}$ \\ Pauline Lambert, Sonia Frasquilho, Laurent Antunes,' Olga Kofanova, Daniel Rohrer, \\ Dana R. Valley, ${ }^{4}$ Alex Blanski, ${ }^{4}$ Scott Jewell, ${ }^{4}$ Helen Moore, ${ }^{3}$ and Fay Betsou ${ }^{1}$
}

\begin{abstract}
Although there are millions of formalin-fixed paraffin-embedded (FFPE) tissue blocks potentially available for scientific research, many are of questionable quality, partly due to unknown fixation conditions. We analyzed FFPE tissue biospecimens as part of the NCI Biospecimen Preanalytical Variables (BPV) program to identify microRNA (miRNA) markers for fixation time. miRNA was extracted from kidney and ovary tumor FFPE blocks (19 patients, cold ischemia $\leq 2$ hours) with $6,12,24$, and 72 hours fixation times, then analyzed using the WaferGen SmartChip platform (miRNA chip with 1036 miRNA targets). For fixation time, principal component analysis of miRNA chip expression data separated 72 hours fixed samples from 6 to 24 hours fixed samples. A set of small nuclear RNA (snRNA) targets was identified that best determines fixation time and was validated using a second independent cohort of seven different tissue types. A customized assay was then developed, based on a set of 24 miRNA and snRNA targets, and a simple "snoRNA score" defined. This score detects FFPE tissue samples with fixation for 72 hours or more, with $79 \%$ sensitivity and $80 \%$ specificity. It can therefore be used to assess the fitness-for-purpose of FFPE samples for DNA or RNA-based research or clinical assays, which are known to be of limited robustness to formalin overfixation.
\end{abstract}

Keywords: miRNA, formalin fixation, quality control, tissue, biobank

\section{Introduction}

$\mathrm{F}$ OR FORMALIN-FIXED PARAFFIN-EMBEDDED (FFPE) tissue, the most critical preanalytical variables are the warm and cold ischemia times, the fixation/stabilization type, the fixation time, and the long-term storage conditions. ${ }^{1,2}$ The fixation process produces covalent cross links; the longer the fixation time the more the cross-linking and the lower the quality of nucleic acids ${ }^{3}$ for sequencing and other molecular studies. ${ }^{4}$

The optimal tissue fixation time, as estimated by RNA integrity electropherogram features and by branched DNA assays, was 12-24 hours in phosphate-buffered formalin, with significant degradation occurring at 36 and 48 hours fixation time and almost complete loss of targeted messenger RNA (mRNA) expression at 72 hours fixation time. ${ }^{5}$ The optimal fixation time for mRNA preservation has been shown to be between 4 and 24 hours, depending on the tissue type and specimen size. ${ }^{6}$
In the context of clinical diagnostic assays, little has been published on the impact of formalin overfixation on FDAapproved or CE-marked assays, performed with nucleic acids from FFPE tissue. Fixation of breast cancer tissue samples of up to 5 days did not affect the results of a clinical diagnostic gene expression assay, based on eight cancer related and 4 reference genes, whereas 10 days of fixation did. ${ }^{7}$ However, clinical diagnostics based on Next Generation Sequencing (NGS) can be biased since the number of deamination variants, $\mathrm{C}: \mathrm{G}>\mathrm{T}: \mathrm{A}$ that are identified by NGS, increases with tissue fixation for $>48$ hours. ${ }^{8}$ Furthermore, for protein analyses by immunohistochemistry (IHC), the American Society of Clinical Oncology/College of American Pathologists guideline for IHC testing of estrogen and progesterone receptors in breast cancer, is that breast tissue specimens must be fixed in $10 \%$ neutralbuffered formalin (NBF) for no $<6$ hours and for not more than 72 hours before processing. Overfixation could lead to

\footnotetext{
${ }^{1}$ IBBL, Dudelange, Luxembourg.

${ }^{2}$ Biostatistics Unit, Institute of Environmental Medicine, Karolinska Institutet, Stockholm, Sweden.

${ }^{3} \mathrm{NCI}$, Bethesda, Maryland.

${ }^{4}$ Van Andel Institute, Grand Rapids, Michigan.
}

(C) Wim Ammerlaan et al., 2018; Published by Mary Ann Liebert, Inc. This Open Access article is distributed under the terms of the Creative Commons Attribution Noncommercial License (http://creativecommons.org/licenses/by-nc/4.0/) which permits any noncommercial use, distribution, and reproduction in any medium, provided the original author(s) and the source are cited. 
false-negative results as a result of excessive protein crosslinking by formaldehyde. The guideline also stipulates that this would particularly be true if there was inadequate antigen retrieval used during the performance of the ER/PgR assays, since usual antigen retrieval protocols are optimized for 24 hours of fixation time. ${ }^{9}$

In the context of research and biomarker discovery, there is evidence that tissue fixation time is a critical factor. Formalin overfixation leads to more DNA damage due to deamination of cytosine bases, which has a detrimental effect on the accuracy of NGS. ${ }^{10}$

Formalin overfixation (1 week vs. 20 hours) generates lowquality labeling and poor comparative genome hybridization profiles in array CGH analyses. ${ }^{11}$ When evaluated in the context of DNA microarray and whole-transcriptome sequencing platforms, liver tissue fixation time of 3 weeks compared to 18 hours resulted in half the RNA yield, increased the RNA input needed for library preparation, and led to lower correlation with frozen samples, detection of fewer differentially expressed genes and deterioration of microarray signal intensity. 2

Although several quality control (QC) tools exist, which allow us to qualify tissue samples, we still do not have an assay that will allow us to identify overfixed tissue samples. ${ }^{13}$

Multiple studies, especially in the cancer field, have suggested that micro RNAs (miRNAs) can be used as diagnostic biomarkers. ${ }^{14,15}$ The advantage of miRNAs lies in their robustness. Due to their small size and the fact that they are protected in protein macromolecular complexes, miRNAs are resistant to degradation and chemical modification in FFPE tissue samples, and correlate with expression levels from corresponding frozen tissue, better than longer mRNAs. ${ }^{16,17}$ Despite the relative robustness of the miRNAs, a miRNA deep-sequencing study on cardiac tissue samples, fixed in formalin for periods of between 5 and 45 days concluded that the miRNA read counts in FFPE specimens are comparable only if the tissue samples are processed under the same fixation conditions. ${ }^{18}$ Apart from the miRNAs, another category of short RNA molecules is the small nuclear RNAs (snRNAs) whose robustness to fixation conditions has not yet been studied.

Our study had two objectives: (1) to evaluate the robustness, not of just a few, but of more than 950 miRNA targets, to different fixation times, and (2) to identify at least one small RNA target that is sensitive to overfixation conditions and therefore could be used as a QC tool to assess overfixed tissue samples, particularly in legacy collections of unknown preanalytics.

\section{Materials and Methods}

\section{Samples used}

Biospecimen Preanalytical Variables (BPV) biospecimens were collected at four Biospecimen Source Sites (BSS) and sent to the centralized BPV biospecimen repository at Van Andel Research Institute where 10 kidney and 9 ovary tumor tissues were selected for this study, with $<2$ hours cold ischemia time, and 10-12 hours processing time (time in the tissue processor). ${ }^{19}$ For each specimen, the BSS prepared a frozen aliquot and FFPE samples with four different fixation times: 6 hours (Sample PREanalytical Code [SPREC] code D), 12 hours (SPREC code E), 23 hours (SPREC code E), and
72 hours (SPREC code G), ${ }^{1,2} 95$ samples in total. These blocks were stored at room temperature until RNA extraction and were used as training set.

From an IBBL biospecimen research collection, six colon/sigmoid, one kidney, one ovary, two uterus, one rectum, six breast, two ileum tissue specimens were selected with 10 minutes to 31 hours ischemic times, and with 12.5 hours processing time. For each specimen, IBBL prepared FFPE samples with three different fixation times: 1215 hours (SPREC code E), 48-52 hours (SPREC code G) and 72-76 hours (SPREC code Z). Fixation times were documented for all 19 specimens, and 57 samples were available for further testing. These blocks were stored at $-20^{\circ} \mathrm{C}$ until RNA extraction and were used as a validation set. All patients signed an informed consent and the study had Ethics Committee approval under UNM IRB protocol 11-279 (University of New Mexico), IRB protocol study ID number 45796 (Emory University), IRB number 0506140 (University of Pittsburg), IRB number H-32145 (Boston Medical Center), and CPP number DC-2010-1145 (France).

\section{RNA extraction and initial qualification}

RNA extraction from all frozen tissue and FFPE samples was performed with miRNeasy Qiagen Kit (Ref. 217504; Qiagen) on a QIAcube platform. RNA quantification was performed by spectrophotometry, and the RNA integrity number (RIN) was determined on an Agilent 2100 Bioanalyzer. The tissue samples of the training set (Van Andel Institute) contained at least $60 \%$ tumor and $<20 \%$ necrosis. The tissue samples of the validation set (IBBL) contained $10 \%-90 \%$ tumor and 0\%-30\% necrosis.

\section{WaferGen SmartChip assay}

$0.5 \mu \mathrm{g}$ of extracted RNA was poly A tailed using Poly(A) Polymerase Tailing Kit (Epicentre) with reverse transcription (RT) buffer from the High-Capacity complementary DNA (cDNA) RT Kit and RNase inhibitor (both Applied Biosystems), for one cycle of 30 minutes at $37^{\circ} \mathrm{C}$, followed by 5 minutes at $70^{\circ} \mathrm{C}$, in a Thermocycler (C1000; Bio-Rad Laboratories). Samples were stored at $4^{\circ} \mathrm{C}$ overnight. Poly Atailed RNA was transcribed to cDNA by a reverse transcription reaction using High-Capacity cDNA Reverse Transcription Kit (Applied Biosystems) with SmartChip miRNA RT primers (WaferGen), running one cycle of 60 minutes at $40^{\circ} \mathrm{C}$, followed by a 5 minutes at $85^{\circ} \mathrm{C}$ inactivation step. cDNA samples were stored at $-80^{\circ} \mathrm{C}$. For quantitative polymerase chain reaction (qPCR), the total cDNA sample was used, mixed with $500 \mu \mathrm{L}$ LightCycler 480 DNA SYBR Green 1 Mix (Roche Applied Science), with $1 \mathrm{mg}$ glycogen (Roche Applied Science), $1 \mu \mathrm{g}$ M13mp18-ssDNA (New England Biolabs), spiked in yeast controls $(2.0 \mu \mathrm{L}$ from Saccharomyces cerevisiae S288c; WaferGen), and made up to $1 \mathrm{~mL}$ with PCR grade $\mathrm{H}_{2} \mathrm{O}$ (Roche Applied Science). Samples were loaded onto a SmartChip Human miRNA Panel v3.0 (WaferGen) with a Multisample Nanodispenser (WaferGen) and processed with a SmartChip Cycler (WaferGen) using Human miRNA v3 Protocol Rev F, or loaded chips were frozen at $-20^{\circ} \mathrm{C}$ until processing. Quadruplicate analysis was performed for each miRNA, fixation time point and tissue type.

To demonstrate the reproducibility of the SmartChip miRNA assay, the Stratagene QPCR Human Reference Total 
RNA (Ref. 750500; Agilent) was run in triplicate, on three different chips, each run on a different day.

\section{WaferGen customized miRNA chip assay}

For high-throughput purposes, a customized WaferGen SmartChip was designed, allowing analysis of 48 samples per SmartChip. This chip contained only the primers for the following 24 targets: HSA-LET-7A, HSA-LET-7D, HSALET-7E, HSA-MIR-103, HSA-MIR-107, HSA-MIR-1251, HSA-MIR-1260, HSA-MIR-154, HSA-MIR-17, HSA-MIR27A, HSA-MIR-320C, HSA-MIR-326, HSA-MIR-339-5P, HSA-MIR-4279, HSA-MIR-720, RNU12, RNU2-1, SCARNA5, SNORA16A, SNORA44, SNORA61, SNORD99,
WGBS-YCF2, and WGBS-YCF3. Each target was printed in quadruplicate per sample loading area. The sample processing for the customized chip was identical to the WaferGen SmartChip Assay as described above; the only difference consisted in dividing the used reagent quantities by 40 per sample. The samples of the validation set were tested on the customized chip.

\section{Data analysis}

Data analysis on the standard miRNA SmartChip. miRNA qPCR profiles were analyzed with the WaferGen qPCR v2.5 software (see Supplementary Table S1 for detailed analysis settings; Supplementary Data are available online at

\section{A Kidney/Time in Fixative}

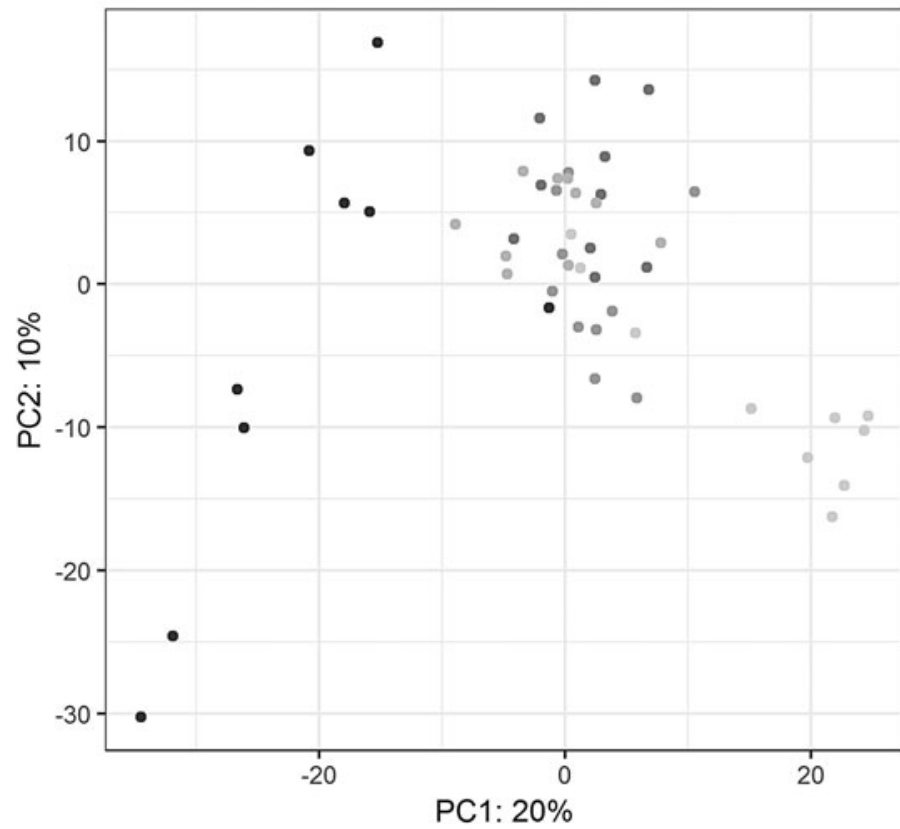

B Ovary/Time in Fixative

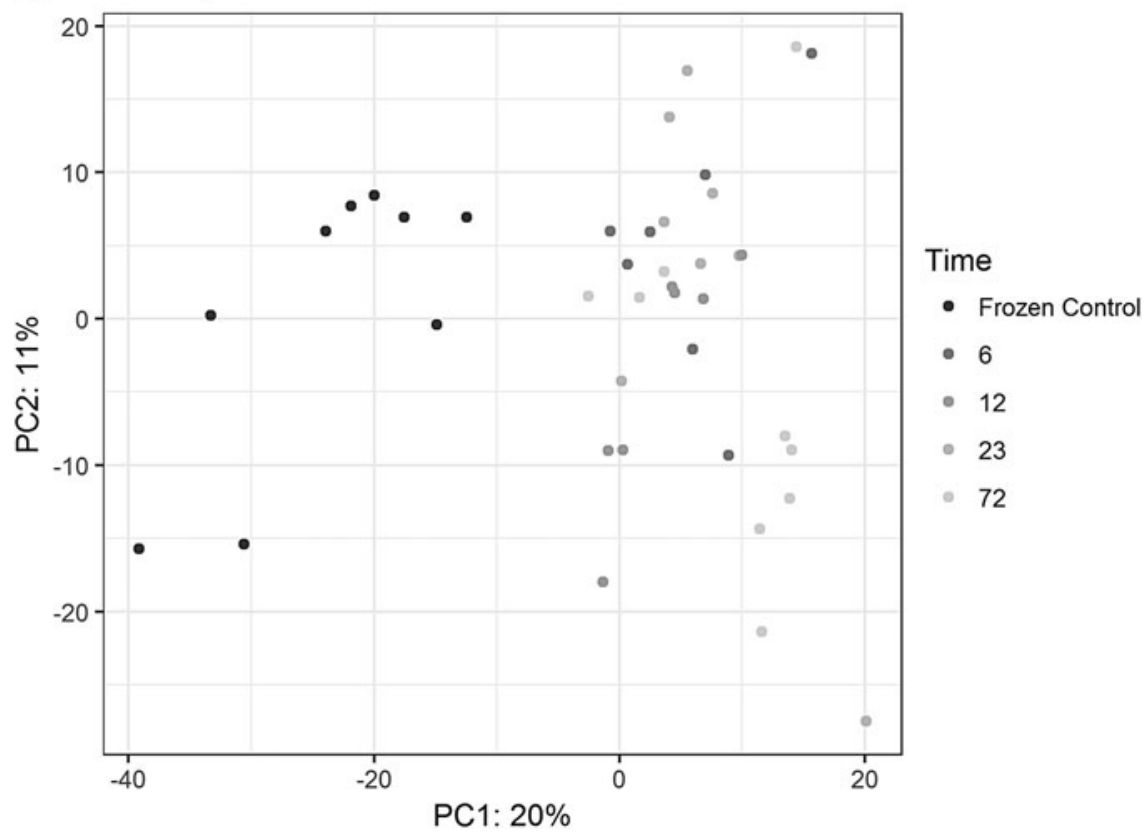

FIG. 1. PCA plot for (A) kidney samples $(n=50)$ and (B) ovary samples $(n=45)$ of the training set. Different dot gray intensities indicate different fixation times $(6,12,23$, and 72 hours). PCA, principal component analysis. 
www.liebertpub.com/bio). Cycle threshold $(\mathrm{Ct})$ values were exported to qBase v 2.6.1 software (Biogazelle) and analyzed by sample grouping according to the fixation times. Global normalization and target scaling to average were used for qBase parameters calculation settings. Calibrated normalized relative quantities (CNRQ) data (global normalization) were exported to Excel (Microsoft) from samples with the following QC criteria: Global normalization factor between 0.3 and 3.33; Reference Targets Stability with an average GNorm $\mathrm{M}<1.0$ and coefficient of variation $(\mathrm{CV})<0.6$. Negative results were replaced by a minimal CNRQ value of 0.050 to avoid artificially decreasing CVs. miRNA variability was expressed in terms of $\mathrm{CV}$ of the CNRQ, and miRNAs were ordered accordingly.

Principal component analysis (PCA) was performed to evaluate miRNA expression patterns of all FFPE experimental

A Control miRNAs - Ovary

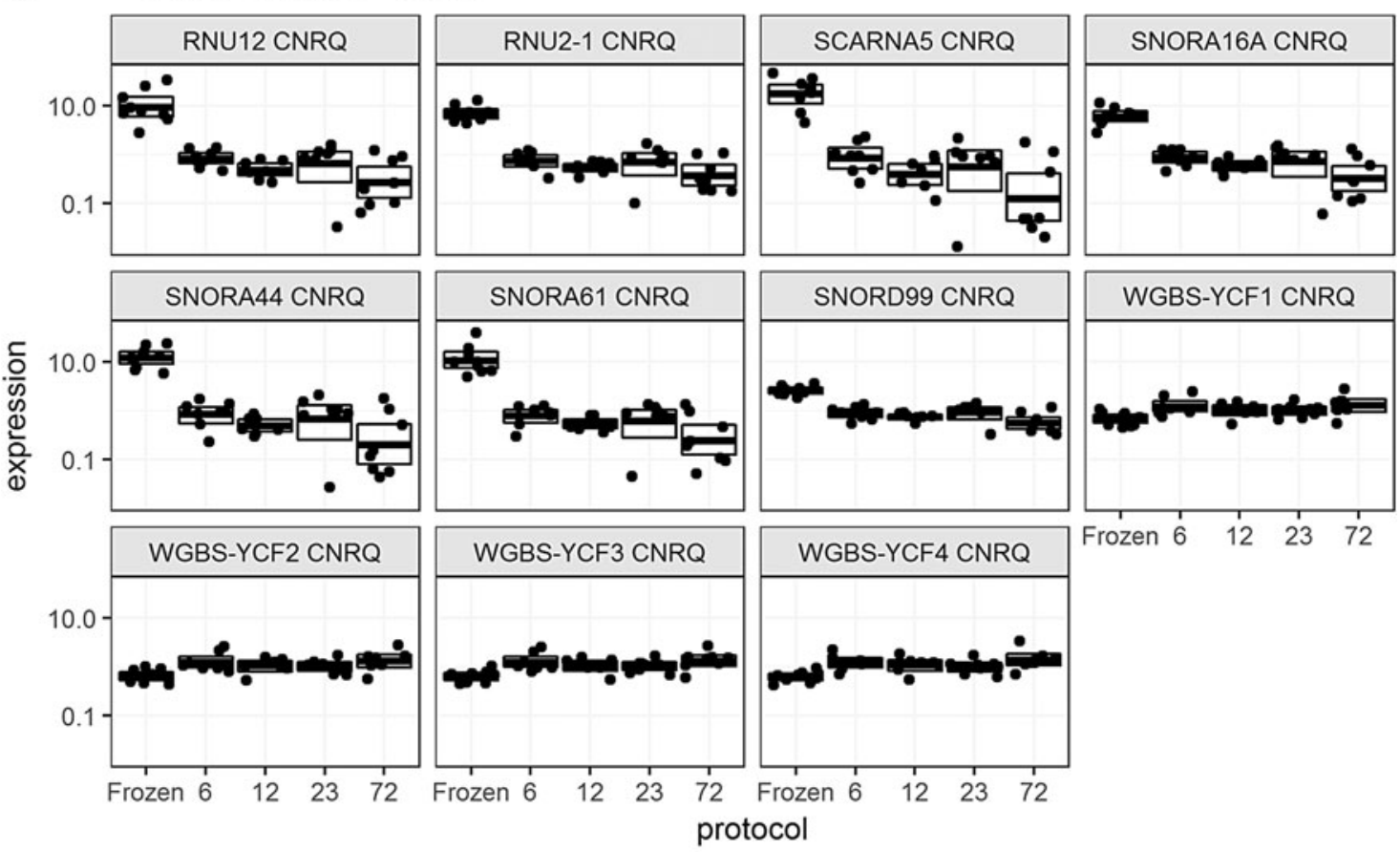

B Control miRNAs - Kidney
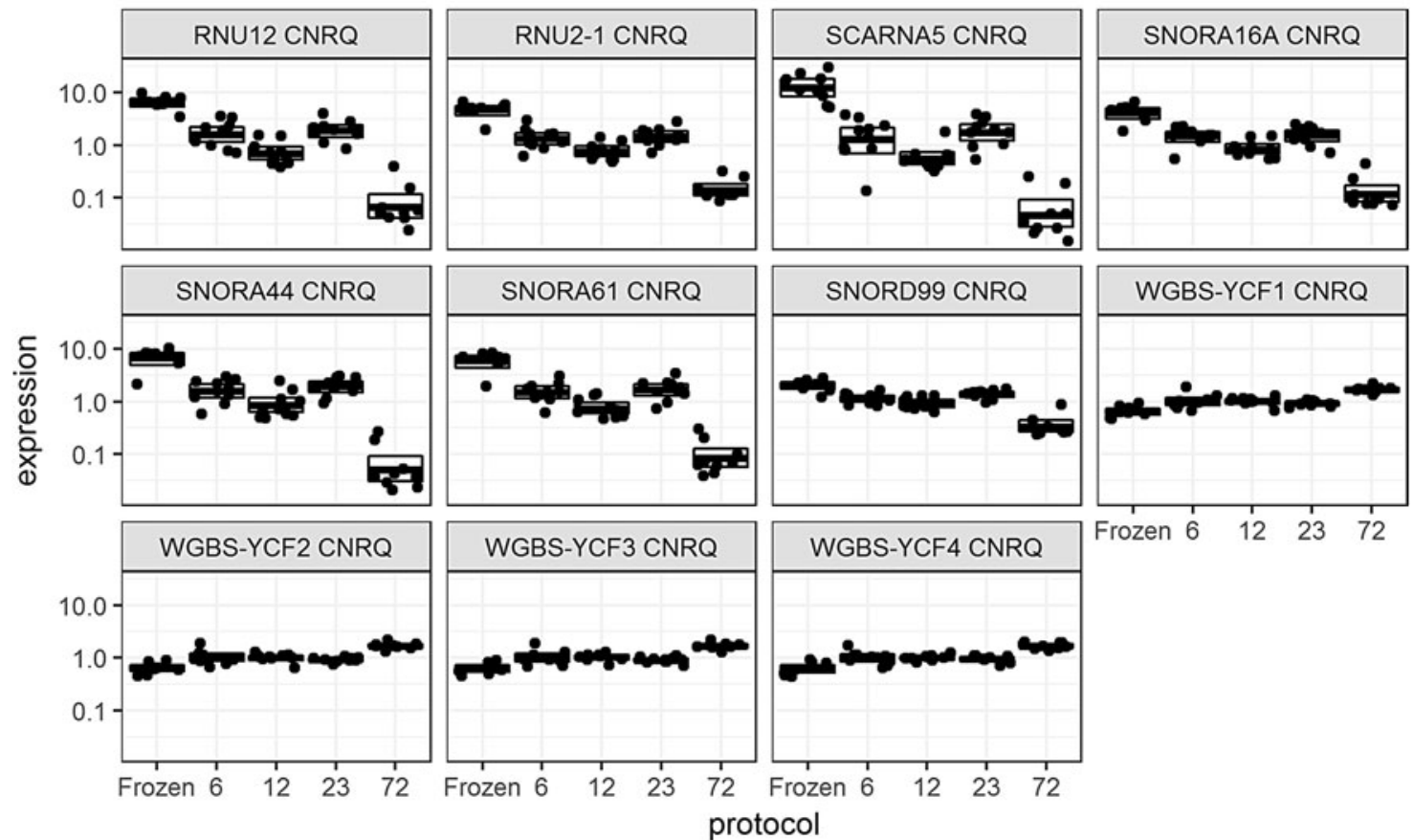

FIG. 2. Box plot analysis of miRNA/snoRNA targets with significant differences in their raw Ct values between frozen samples and samples fixed for different fixation times (fixation times indicated on the horizontal axis), in the training set of kidney (A) and ovary (B) tissue specimens. WGBS-YCF1-4 are the yeast internal controls on the WaferGen standard SmartChip. Ct, cycle threshold; miRNA, microRNA; snoRNA, small nucleolar RNA. 
groups relative to each other. For each miRNA, linear mixed effects models with random intercepts were used to estimate differences in log expression by protocol, while accounting for the within-sample correlation due to specimens coming from the same patient. The model contained five parameters, the intercept and four parameters for each of the preanalytical conditions (fixation for $6,12,23$, and 72 hours). We used the likelihood ratio test for the global hypothesis that any of the four parameters differ from 0 . This is a test of whether there are any differences in average expression among the preanalytical conditions tested, including the frozen control, without assuming linear relationships. We repeated this model excluding the frozen control, thereby testing for any differences in miRNA expression among the fixation times. Box plots were calculated, showing the median and $95 \%$ confidence intervals for the median. The accuracy of the expression scores for predicting fixation times were assessed using receiver operating characteristic (ROC) curve analysis, in which we estimated sensitivity, specificity, and the area under the ROC curve. To select an optimal cutoff from the ROC curve, we calculated the point with the minimum distance to the upper-leftmost corner $(0,1)$.

Analyses were performed using $\mathrm{R}$ version 3.3.2 ( $\mathrm{R}$ Foundation for Statistical Computing, Vienna, Austria).

Data analysis for the selection of the targets of the customized chip. For detection of the highly expressed miRNAs, either stable or variable, the CVs of the CNRQ values for each fixation time and tissue type were ranked together with the mean of the corresponding replicate nonnormalized $\mathrm{Ct}$ values. The miRNA targets were selected based on detection in more than $95 \%$ of the samples and a quantification level of $<22 \mathrm{Cts}$. A panel of 15 fixation-stable miRNA (CNRQ CV <20\%), 7 fixation-unstable small RNAs (CNRQ CV >50\%), and 2 yeast control targets were selected for the customized chip panel.

\section{Results}

\section{Reproducibility of the SmartChip miRNA assay}

Global normalization of the qPCR Human Reference Total RNA, run in triplicate, on three different chips, resulted in normalization factors of $0.95,1.02$, and 1.04, respectively. The RNU12, RNU2-1, SCARNA5, SNORA16A, SNORA44, SNORA61, and SNORD99 were used as reference targets in the WaferGen miRNA SmartChip. The stability of each of these reference targets, as expressed in GNorm, was in the range of $0.07-0.14$, and the $\mathrm{CV}$ ranged from $3 \%$ to $10 \%$, both parameters being within the qBase acceptance range of variance. The coefficient of determination $\left(R^{2}\right)$ between every combination of two replicates ( 1 vs. 2,1 vs. 3 , and 2 vs. 3 ) was 0.98-0.99 (Supplementary Fig. S1).

\section{Training set; miRNA robustness to fixation time}

For the ovary tissue, the average RIN obtained from 36 FFPE samples was 2.3 (standard deviation [SD] 0.6), while the average RIN obtained from the 9 corresponding frozen samples was 7.8 (SD 2.8). For the kidney tissue, the average RIN obtained from 40 FFPE samples was 2.8 (SD 1.0), while the average RIN obtained from the 10 corresponding frozen samples was 6.7 (SD 1.7).

The standard miRNA WaferGen SmartChip was used and two samples were excluded due to technical errors during manipulation.

The $F$ test was performed for the global hypothesis that any of the four conditions (frozen, fixed for 6, 12, 23, and 72 hours) were significantly different. When the frozen samples were included in the analysis, in both ovary and kidney tissue, there were some marked differences in miRNA expression between FFPE samples and frozen control samples. On average, the fold difference in expression between frozen and FFPE was $98.7 \%$ in kidney and $117.3 \%$ in ovary tissue. For some miRNAs, the FFPE samples had lower expression levels, while for other miRNAs, FFPE samples had higher expression levels than their frozen counterparts. In kidney tissue, there were some differences in miRNA expression, between fixation times, among FFPE samples. On average, the fold difference in expression levels, between 72 and 6 hours fixation was $97.4 \%$. We did not find many significant differences in expression levels among ovary FFPE samples. All the $p$-values are shown in Supplementary Table S2. PCA plot analysis showed good separation not only between FFPE and frozen samples but also between 72 hours fixation time (over-fixed samples) and 6, 12, and 23 hours fixation times (Fig. 1).

\section{Identification of miRNAs sensitive to overfixation conditions}

After classification of all the miRNAs and small nucleolar RNAs (snoRNAs) based on CNRQ variability (CV \%), we found that the most unstable targets were SCARNA5, SNORA16A, SNORA44, and SNORA61 (Fig. 2).

We calculated a score ("snoRNA CNRQ score") that is the sum of the logCNRQ values for SCARNA-5, SNORA16A, SNORA-44, and SNORA-61 (snoRNA CNRQ score = $\log$ CNRQ SCARNA5 + $\log$ CNRQ SNORA16A + $\log$ CNRQ SNORA44 + logCNRQ SNORA61). Based on this score, there was a clear distinction between samples with fixation time corresponding to SPREC codes D and E (6-12 hours)

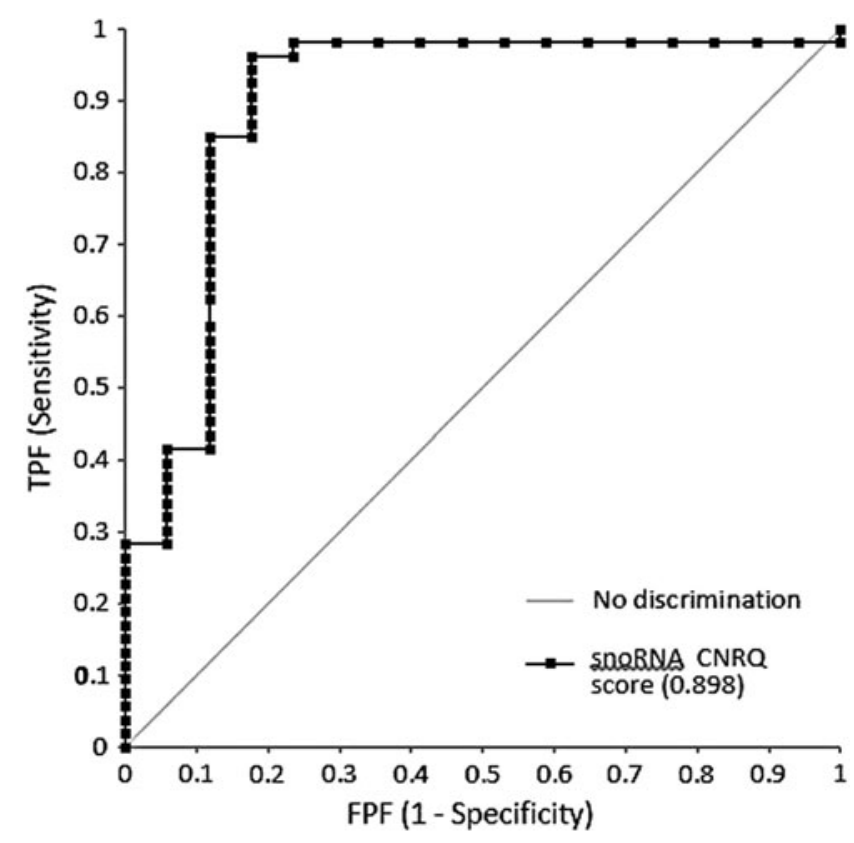

FIG. 3. ROC curve for the ovary and kidney tissue samples (training set, including samples of different fixation times, but not the frozen samples, $n=78$ ), analyzed on the WaferGen standard SmartChip with the "snoRNA CNRQ score" analysis (snoRNA CNRQ score $=\log$ CNRQ SCARNA5+ $\log$ CNRQ SNORA16A + $\log$ CNRQ SNORA44+ $\log$ CNRQ SNORA61). CNRQ, calibrated normalized relative quantities; ROC, receiver operating characteristic. 
FIG. 4. Boxplots of CNRQ scores of representative examples of unstable (A-C) and stable (D, E) targets on the WaferGen standard SmartChip, with RNA extracted from 54 tissue samples, collected in IBBL (validation set).
A

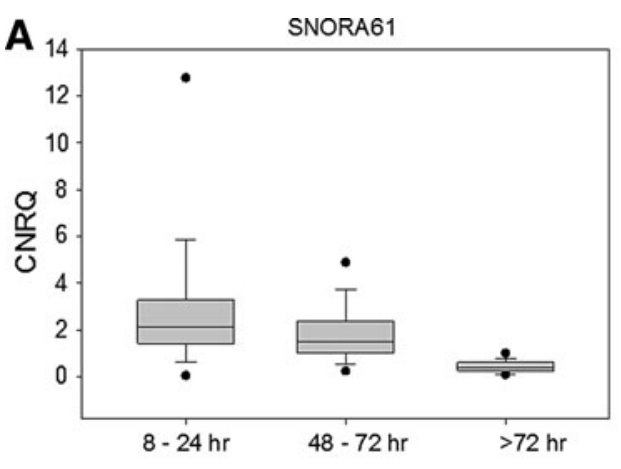

\section{C}
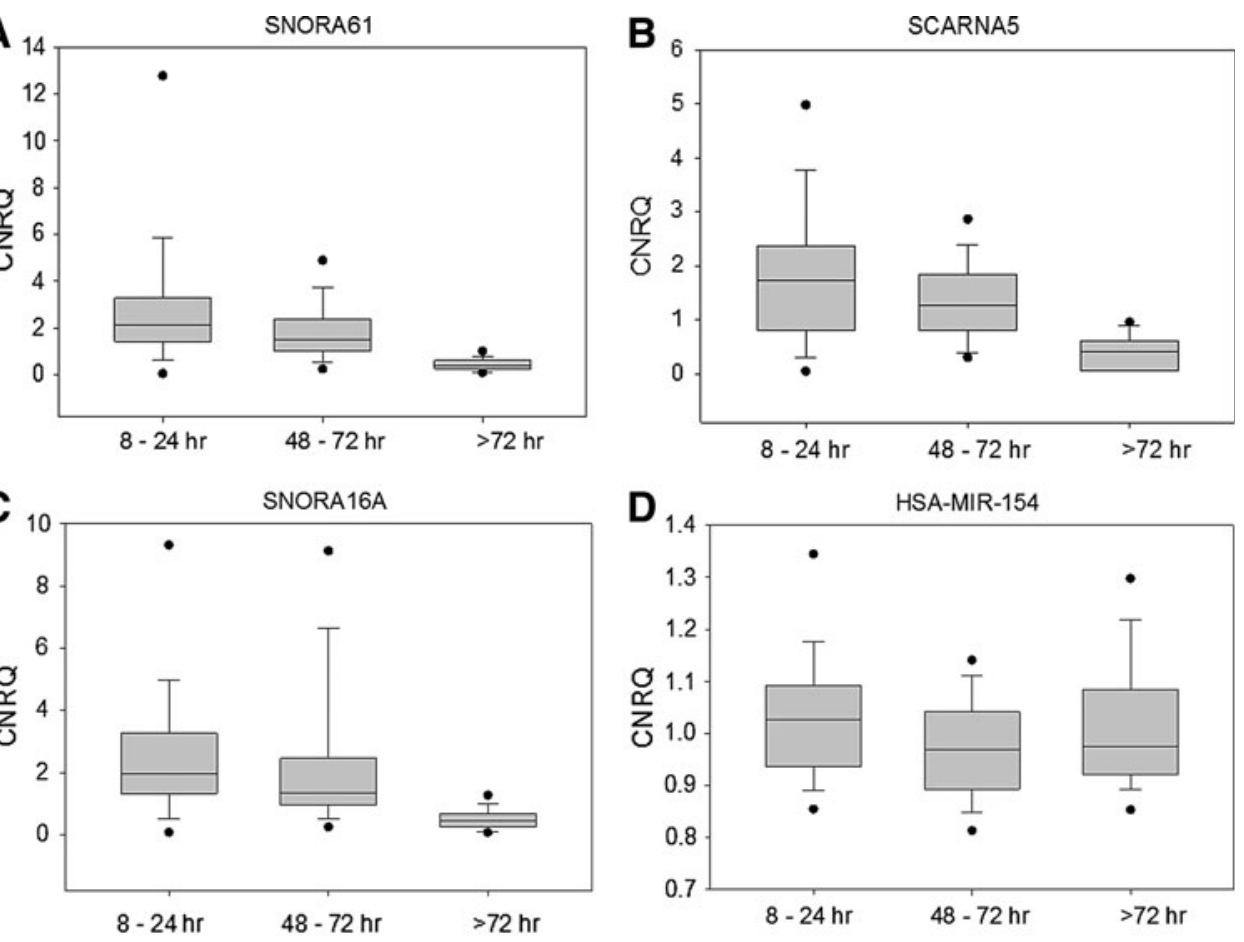

D 1

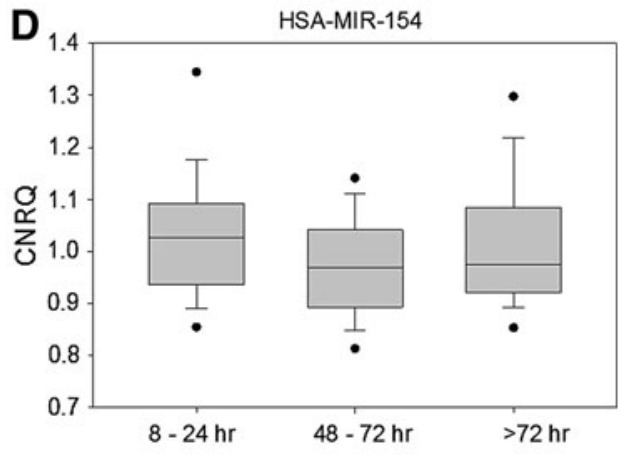

E

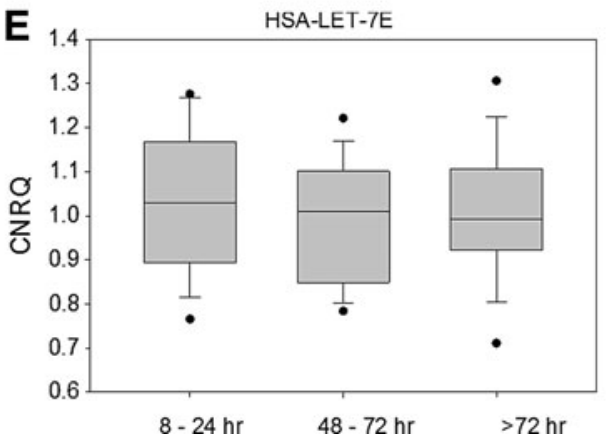

and samples with fixation time corresponding to SPREC code $\mathrm{G}$ (72 hours). ROC curve analysis based on this score showed a sensitivity of $85 \%$ and a specificity of $88 \%$ (area under the curve $[\mathrm{AUC}]=0.898$ ) in the detection of FFPE tissue samples that were fixed for 72 hours versus those that were fixed for 6 , 12, or 23 hours (Fig. 3).

\section{Validation set}

The "snoRNA CNRQ score" was applied to 19 specimens of different tissue types, collected in IBBL, each subjected to 3 fixation times; 8-24 hours (SPREC code E), 48-72 hours (SPREC code $\mathrm{G}$ ), and $>72$ hours (SPREC code Z). Four samples failed on the WaferGen SmartChip assay; one sample with $\mathrm{E}$, one with $\mathrm{Z}$, and two with $\mathrm{G}$ fixation time points. The sensitivity of the snoRNA targets to overfixation was confirmed (Fig. 4), and ROC curve analysis, based on the "snoRNA CNRQ score," showed a sensitivity of $88 \%$ and a specificity of $91 \%$ in the detection of FFPE tissue samples with $\geq 72$ hours fixation time (Fig. 5).

\section{Customized WaferGen miRNA chip assay}

The median CNRQ values of tissue samples with fixation times corresponding to SPREC codes E, G, and Z, for 24 targets, which were selected for the customized WaferGen chip, are shown in Supplementary Figure S2. There were two groups of targets (apart from the two yeast controls): one group, including the HSA-LET-7A, HSA-LET-7D, HSA-LET-7E, HSA-MIR-103, HSA-MIR-107, HSA-MIR1251, HSA-MIR-1260, HSA-MIR-154, HSA-MIR-17, HSA-MIR-27A, HSA-MIR-320C, HSA-MIR-326, HSAMIR-339-5P, HSA-MIR-4279, and HSA-MIR-720, with overall stable CNRQ values, and one group, including the RNU12, RNU2-1, SCARNA5, SNORA16A, SNORA44, SNORA61, and SNORD99, with high CNRQ values at standard fixation conditions and low CNRQ values at overfixation conditions.

Eight sets of 3 samples (24 samples total) from the IBBL collection were tested on the customized WaferGen chip. One set of samples was excluded from further analysis for not having a sample fixed for more than 72 hours. A coefficient of determination $R^{2}$ of 0.74 was observed between the $\mathrm{Ct}$ values on the standard miRNA SmartChip and on the customized chip (Supplementary Fig. S2B). On the customized chip, a simple "snoRNA score" was calculated by subtracting the sum of the Ct values of HSA_MIR_720, HSA-MIR-1260, and HSA-LET-7A from the sum of the $\mathrm{Ct}$ values of SNORD99, SNORA61, SNORA44, SNORA16A, and SCARNA5; whereas the "snoRNA CNRQ score" requires 


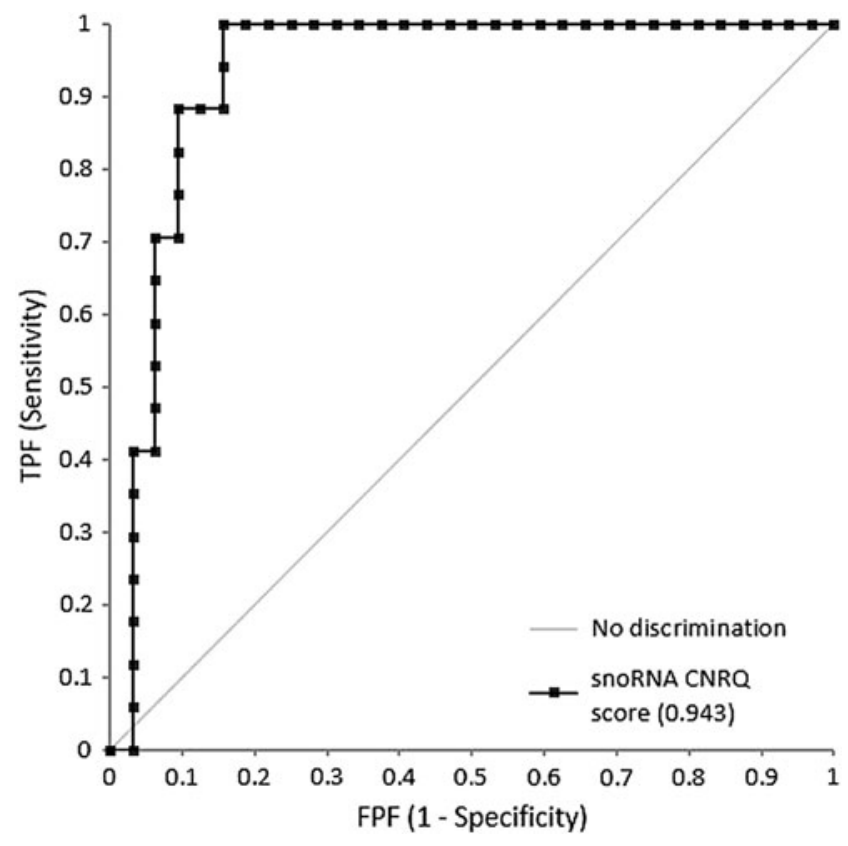

FIG. 5. ROC curve for the colon/sigmoid, kidney, ovary, uterus, rectum, breast, and ileum tissue samples (validation set, $n=57)$, based on the "snoRNA CNRQ score" analysis ( snoRNA CNRQ score $=\log$ CNRQ SCARNA5 $+\log$ CNRQ SNORA16A $+\log$ CNRQ SNORA44 + $\log$ CNRQ SNORA61) on the WaferGen standard SmartChip.

the whole miRNA SmartChip, and therefore, only one sample can be tested per run, the simplified "snoRNA score" requires only 24 targets and 40 samples can be tested per run. The repeatability of the "snoRNA score," validated on 37 technical replicates had a CV of 3.4\% (data not shown). ROC curve analysis gave us an AUC of 1.000 for both distinctions between fixation time corresponding to SPREC codes $G$ versus $Z$ and between fixation time SPREC codes E versus $Z$ (data not shown).

This snoRNA score $\left(\mathrm{Ct}_{\text {SNORD99 }}+\mathrm{Ct}_{\text {SNORA61 }}+\mathrm{Ct}_{\text {SNORA44 }}\right.$ $+\mathrm{Ct}_{\text {SNORA16A }}+\mathrm{Ct}_{\text {SCARNA5 }}-\mathrm{Ct}_{\text {HSA_MIR_720 }}-\mathrm{Ct}_{\text {HSA-MIR-1260 }}$ $\left.-\mathrm{Ct}_{\mathrm{HSA}-\mathrm{LET} \text {-7A }}\right)$ was then applied to the raw data obtained by our initial FFPE training set of two tissue types (50 kidney samples, 45 ovary samples) and the whole validation set of seven different tissue types. For the training set, the new ROC curve analysis, based on the snoRNA score, gave AUC 0.989 for the distinction between 6,12 , and 23 hours versus 72 hours fixation time of kidney samples and AUC 0.728 for ovary samples. When all the kidney and ovary samples were analyzed together, the ROC curve analysis, based on the snoRNA score, gave AUC 0.931 with an optimal cutoff value 67. This corresponds to a sensitivity of $83.3 \%$ and specificity of $82.5 \%$ for the detection of overfixed samples.

In the validation set of 53 samples, the distinction between SPREC code E and G versus Z, showed AUC 0.847, with the optimal cutoff value 85 , corresponding to a sensitivity of $83.3 \%$ and specificity of $84.4 \%$ for the detection of overfixed samples (Fig. 6).

Finally, we defined the optimal cutoff for the snoRNA score, by analyzing the samples of both the training and the validation sets together. The diagnostic cutoff was 79 , with AUC 0.854 corresponding to a sensitivity of $78.9 \%$ and specificity of $80.4 \%$ for the detection of overfixed samples (Fig. 6).

\section{Discussion}

The Qiagen miRNeasy FFPE Kit used in this study has been shown to be the most effective extraction kit for FFPE miRNAs. ${ }^{20}$ The choice of the WaferGen platform for detecting differences between miRNAs was based on published data demonstrating very high sensitivity and reproducibility. ${ }^{21}$ The data analysis pipeline that we used combines the power of both the WaferGen and the qBase analysis programs. While it has recently been shown that versions of miRBase published after miRBase16 contain many false miRNAs, which are RNA degradation products, ${ }^{22}$ this issue has no effect on our study since the WaferGen miRNA chip composition is based on miRBase 16.

This study is the first to have evaluated the stability of 1036 miRNA targets to fixation conditions by RT-qPCR. The results show a global and uniform robustness of the miRnome to NBF fixation times up to 72 hours. This can be explained by the uniform molecular structure of the miRNAs and confirms the reliability of FFPE miRNA profiling. ${ }^{23}$

Unsurprisingly, paraffin embedding and formalin fixation induced lower miRNA expression levels, compared to frozen samples, confirming previously published comparisons. ${ }^{24}$ Tissue heterogeneity between frozen and FFPE tissue counterparts may always be the origin of some degree of variability.

The impact of the duration of formalin fixation on global miRNA expression in colorectal and pancreatic tissue has been studied, and 3- or 6-day fixation induced a global reduction in miRNA expression by -1.06 and $-0.64 \mathrm{C}_{\mathrm{q}}$ relative to 2-day fixation conditions, respectively. ${ }^{24}$ Fixation times of 1-5 days did not have a significant impact on the expression levels of miRNA-16 and RNU6B in colorectal FFPE samples. ${ }^{25}$ Our results are in line with previously published results showing a minimal impact of formalin fixation time on miRNAs overall.

Having identified and validated the signature of fixation time-sensitive snRNAs on the WaferGen platform, we tried to transfer the assay to a standard TaqMan platform. We used custom designed TaqMan Small RNA assays for targeted SNORAs (Ref. 4398987; Applied Biosystems). The results of this TaqMan assay confirmed the trend of the snoRNA targets Ct increasing with fixation time, however, the sensitivity of this platform was lower than the sensitivity of the WaferGen platform (materials/methods/results not shown), probably due to different chemistries between the two platforms.

The explanation of the relatively higher impact of fixation time on snRNAS than on miRNAs is probably structural. Mature miRNAs are $\sim 22$ nucleotides (nt) long, while snRNAs are slightly longer (80-200 nt). Different structure, different association with proteins and/or different GC contents may explain the differential stability of snRNAs. Indeed, it has recently been shown that miRNAs with GC content lower than $40 \%$ are more degraded than GC-rich miRNAs in FFPE cardiac tissue samples. ${ }^{18}$ Interestingly, the percent GC content of SNORA-16A, SCARNA-5, SNORA-44, and SNORA-61 is in the range of $45 \%-51 \%$, which is lower than the average percent GC content of most miRNAs. It has recently been suggested that at least one snRNA (RNU6B) in prostate FFPE samples degrades more rapidly than other miRNAs. ${ }^{26}$ The snoRNA and the small Cajal body-specific RNAs (scaRNAs) are 48-250nt long, have either a CDBox or a 

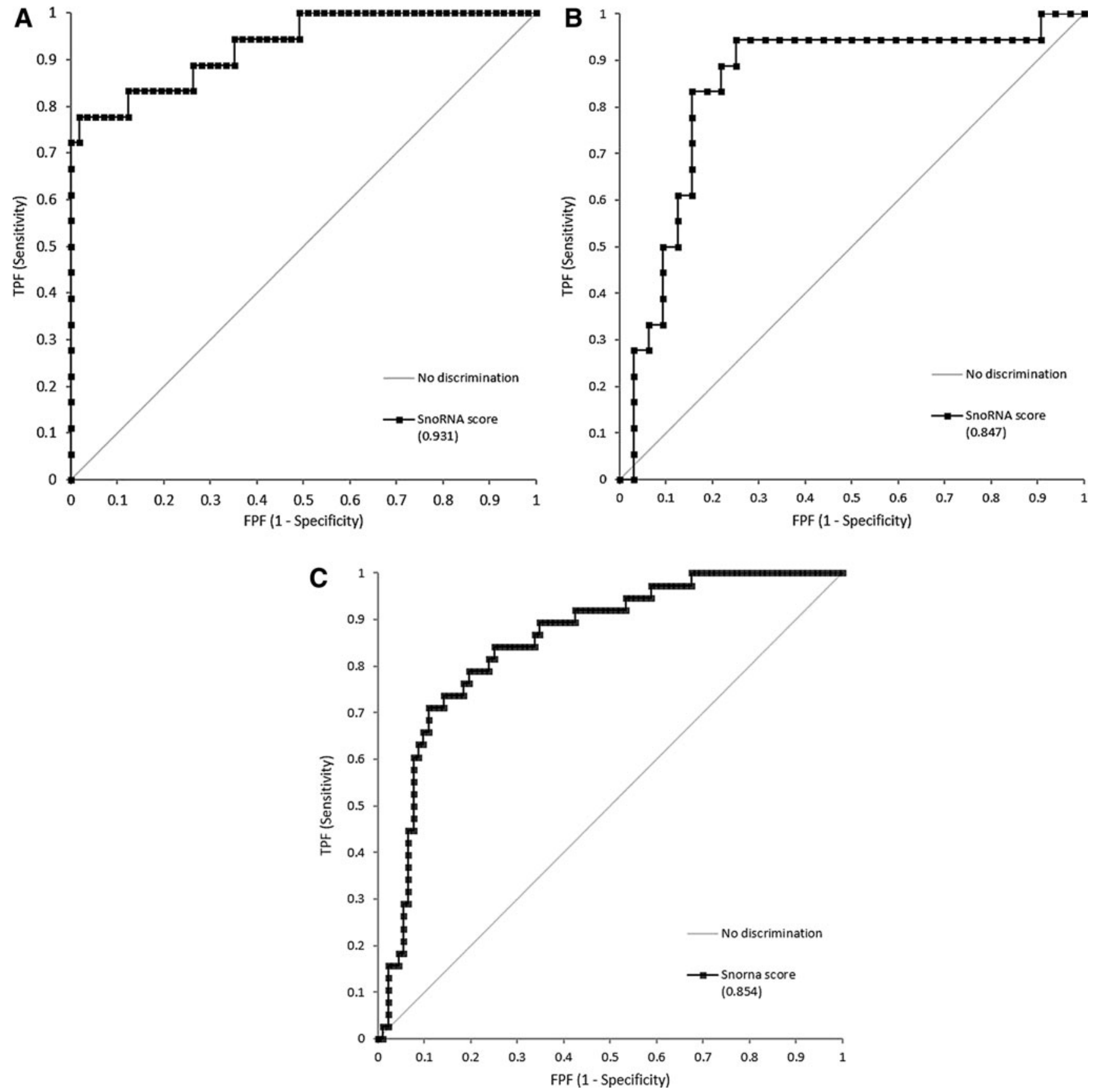

FIG. 6. ROC curve on the samples of the training set, $n=95$ (A), the samples of the validation set, $n=57$ (B), and all the samples of both sets analyzed together, $n=152$ (C), based on the simple snoRNA score on the WaferGen-customized miRNA chip.

HAcaBox secondary structure, and are often associated with the abundant nucleolar protein fibrillarin. ${ }^{27}$ Extended fixation times may induce extensive cross-links among the snoRNAs, scaRNAs, and their associated proteins, rendering their extraction more inefficient than extraction of miRNAs.

A limitation of the snoRNA score assay is that, for optimal sensitivity and specificity, the assay should be performed on the WaferGen platform. The customized chip contains 24 targets, of which 8 are used in the calculation of the snoRNA score. The other 16 targets are used in the establishment of the analytical run acceptance criteria: average yeast internal control $\mathrm{Ct}$ between 17 and 21 and average panel $\mathrm{Ct}$ between
17 and 23. An advantage of the snoRNA score assay is the relatively low amount of RNA required ( $25 \mathrm{ng}$ of total RNA).

Results on the stability of miRNAs in FFPE blocks, stored at room temperature for long periods of time, are conflicting. Some studies suggest that the FFPE block age, when FFPE blocks are stored at room temperature, is a major factor of decrease of all transcript levels, including miRNAs. This has been suggested in studies with myometrium tissue samples ${ }^{28}$ and more recently in a study with prostate tissue samples. ${ }^{26}$ However, in these RT-qPCR studies, different groups of samples from the different time periods were used, and therefore one cannot exclude the possibility of another confounding factor, which may have contributed to the lower 
levels of miRNA targets found in the older samples. Bovell et al. have not found any significant effect of block age on six miRNA targets in colorectal tissue blocks stored for 28 years. $^{29}$ On the contrary, Boisen et al. found a significant effect of $-0.05 \mathrm{Cq} /$ year of sample age on global miRNA expression in pancreatic tissue of up to 30 years old, but not in colorectal tissue of up to 12 years old. ${ }^{24}$ Taking this kinetic assumption, the snoRNA score can safely be used in FFPE samples, fixed with $\mathrm{NBF}$, and stored either at room temperature for 10 years or at $-20^{\circ} \mathrm{C}$ for much longer periods.

In our study, the cold ischemia time was constant in the training set of samples and variable in the validation set. The processing time (time in tissue processor) was 1012.5 hours for all samples. It has been shown that tissue degradation due to ischemia time or freeze thawing has minimal or no impact on miRNAs, ${ }^{30,31}$ and therefore, the snoRNA score assay can be applied irrespective of these preanalytical variables. More specifically, Borgan et al. have shown that there are only 54 miRNas whose expression levels are influenced by tissue ischemia time and snRNAs are not part of them ${ }^{30}$; the snoRNA score therefore is not influenced by ischemia, but only by fixation time.

In conclusion, there is no significant impact of formalin fixation time on miRNA expression levels overall. Furthermore, the snoRNA score assay, validated on seven different tissue types, can identify FFPE tissue samples (of at least the seven tissue types tested) with 72 hours or longer fixation times. This assay fills an important gap in the toolbox of biospecimen qualification assays and enables quality stratification of legacy FFPE tissue collections of unknown preanalytics. It allows the user to categorize the FFPE samples in at least two quality classes, based on fixation time. The user can take this information into consideration when analyzing the results from his research. Hence, the snoRNA score assay will ensure fitnessfor-purpose of FFPE samples for DNA- or mRNA-based applications and increase research reproducibility.

\section{Acknowledgments}

We are thankful to Monica Marchese and Lieke Mevis for excellent assistance with ROC plots and article formatting. This research did not receive any specific grant from funding agencies in the public, commercial, or not-for-profit sectors.

\section{Author Disclosure Statement}

None of the authors has a conflict of interest that could compromise the integrity of this project.

\section{References}

1. Betsou F, Lehmann S, Ashton G, et al. Standard preanalytical coding for biospecimens: Defining the sample PREanalytical code. Cancer Epidemiol Biomarkers Prev 2010;19:1004-1011.

2. Lehmann S, Guadagni F, Moore H, et al. Standard preanalytical coding for biospecimens: Review and implementation of the Sample PREanalytical Code (SPREC). Biopreserv Biobank 2012;10:366-374.

3. Foss RD, Guha-Thakurta N, Conran RM, et al. Effects of fixative and fixation time on the extraction and polymerase chain reaction amplification of RNA from paraffin- embedded tissue: Comparison of two housekeeping gene mRNA controls. Diagn Mol Pathol 1994;3:148-155.

4. Macabeo-Ong M, Ginzinger DG, Dekker N, et al. Effect of duration of fixation on quantitative reverse transcription polymerase chain reaction analyses. Mod Pathol 2002;15: 979-987.

5. Chung J-Y, Braunschweig T, Williams R, et al. Factors in tissue handling and processing that impact RNA obtained from formalin-fixed, paraffin-embedded tissue. J Histochem Cytochem 2008;56:1033-1042.

6. van Maldegem F, de Wit M, Morsink F, et al. Effects of processing delay, formalin fixation, and immunohistochemistry on RNA recovery from formalin-fixed paraffinembedded tissue sections. Diagn Mol Pathol 2008;17: 51-58.

7. Poremba C, Uhlendorff J, Pfitzner BM, et al. Preanalytical variables and performance of diagnostic RNA-based gene expression analysis in breast cancer. Virchows Arch 2014; 465:409-417.

8. Kim S, Park C, Ji Y, et al. Deamination effects in formalinfixed, paraffin-embedded tissue samples in the era of precision medicine. J Mol Diagn 2017;19:137-146.

9. Hammond MEH, Hayes DF, Dowsett M, et al. American Society of Clinical Oncology/College of American Pathologists guideline recommendations for immunohistochemical testing of estrogen and progesterone receptors in breast cancer. Arch Pathol Lab Med 2010;134:907-922.

10. Do H, Dobrovic A. Sequence artifacts in DNA from formalin-fixed tissues: Causes and strategies for minimization. Clin Chem 2015;61:64-71.

11. Ghazani A, Arneson N, Warren K, et al. Limited tissue fixation times and whole genomic amplification do not impact array CGH profiles. J Clin Pathol 2006;59:311-315.

12. Webster AF, Zumbo P, Fostel J, et al. Mining the archives: A cross-platform analysis of gene expression profiles in archival formalin-fixed paraffin-embedded tissues. Toxicol Sci 2015;148:460-472.

13. Betsou F, Bulla A, Cho SY, et al. Assays for qualification and quality stratification of clinical biospecimens used in research: A technical report from the ISBER Biospecimen Science Working Group. Biopreserv Biobank 2016;14: 398-409.

14. Fabbri M. miRNAs as molecular biomarkers of cancer. Exp Rev Mol Diagn 2010;10:435-444.

15. Klopfleisch R, Weiss A, Gruber A. Excavation of a buried treasure-DNA, mRNA, miRNA and protein analysis in formalin fixed, paraffin embedded tissues. Histol Histopathol 2011;26:797-810.

16. Doleshal M, Magotra AA, Choudhury B, et al. Evaluation and validation of total RNA extraction methods for microRNA expression analyses in formalin-fixed, paraffinembedded tissues. J Mol Diagn 2008;10:203-211.

17. Liu A, Tetzlaff MT, VanBelle P, et al. MicroRNA expression profiling outperforms mRNA expression profiling in formalin-fixed paraffin-embedded tissues. Int J Clin Exp Pathol 2009;2:519.

18. Kakimoto Y, Tanaka M, Kamiguchi H, et al. MicroRNA stability in FFPE tissue samples: Dependence on GC content. PloS One 2016;11:e0163125.

19. U.S. Department of Health and Human Services. NIH. National Cancer Institute. Biospecimen Pre-analytical Variables (BPV) Program. https://biospecimens.cancer.gov/programs/ bpv/bpv_page2.asp (Last accessed September 4, 2018).

20. Howe K. Extraction of miRNAs from formalin-fixed paraffinembedded (FFPE) tissues. In: Sweta R, ed. MicroRNA 
Profiling: Methods and Protocols. New York: Humana Press; 2017: 17-24.

21. Mestdagh P, Hartmann N, Baeriswyl L, et al. Evaluation of quantitative miRNA expression platforms in the microRNA quality control (miRQC) study. Nat Methods 2014;11:809-815.

22. Ludwig N, Becker M, Schummann T, et al. The severe impact of RNA integrity on microRNA abundance. Nat Sci Rep 2017;7:5162.

23. Tanić M, Yanowski K, Andrés E, et al. miRNA expression profiling of formalin-fixed paraffin-embedded (FFPE) hereditary breast tumors. Genom Data 2015;3:75-79.

24. Boisen MK, Dehlendorff C, Linnemann D, et al. MicroRNA expression in formalin-fixed paraffin-embedded cancer tissue: Identifying reference microRNAs and variability. BMC Cancer 2015; 15:1024.

25. Xi Y, Nakajima G, Gavin E, et al. Systematic analysis of microRNA expression of RNA extracted from fresh frozen and formalin-fixed paraffin-embedded samples. RNA 2007; 13:1668-1674.

26. Peskoe SB, Barber JR, Zheng Q, et al. Differential longterm stability of microRNAs and RNU6B snRNA in 12-20 year old archived formalin-fixed paraffin-embedded specimens. BMC Cancer 2017;17:32.

27. Balakin AG, Smith L, Fournier MJ. The RNA world of the nucleolus: Two major families of small RNAs defined by different box elements with related functions. Cell 1996;86: 823-834.

28. Szafranska AE, Davison TS, Shingara J, et al. Accurate molecular characterization of formalin-fixed, paraffinembedded tissues by microRNA expression profiling. J Mol Diagn 2008;10:415-423.

29. Bovell L, Shanmugam C, Katkoori VR, et al. miRNAs are stable in colorectal cancer archival tissue blocks. Front Biosci (Elite Ed) 2012;4:1937-1940.

30. Borgan E, Navon R, Vollan HKM, et al. Ischemia caused by time to freezing induces systematic microRNA and mRNA responses in cancer tissue. Mol Oncol 2011;5: 564-576.

31. Peiro-Chova L, Pena-Chilet M, Lopez-Guerrero JA, et al. High stability of microRNAs in tissue samples of compromised quality. Virchows Arch 2013;463:765-774.

Address correspondence to:

Wim Ammerlaan, MS

$I B B L$

1, rue Louis Rech L-3555 Dudelange Luxembourg

E-mail: wim.ammerlaan@ibbl.lu 